\title{
Coal and Rock Recognition at Fully Mechanized Caving Mining Face Based on Variational Mode Decomposition and PSO-SVM
}

\author{
Xue Guanghui ${ }^{1}$, Jiao Yabo $^{2 *}$ and Hu Baohua ${ }^{3}$ \\ ${ }^{1,2,3}$ School of Mechanical Electronic \& Information Engineering, China University \\ of Mining and Technology(Beijing), Beijing 100083, China \\ ${ }^{1} x g h @$ cumtb.edu.cn, ${ }^{2}$ Jiaoyabo1992@163.com, ${ }^{3}$ hubaohua322@163.com
}

\begin{abstract}
At fully mechanized caving mining face, the degree of coal falling is mainly determined by worker's experience, which can easily lead to the problem of "over caving" or "under caving". Aiming at the problem, this paper put forward a method of coal and rock recognition based on variational mode decomposition and PSO-SVM. This paper carried out the top coal and rock caving experiment and obtained the vibration signal of the hydraulic support tail beam under the different working conditions of coal falling and rock falling. We analyzed the signal with the method of variational mode decomposition after the number of Intrinsic Mode functions confirmed. Through comparative analysis, we found that there are many differences in modal function energy percentage among different work conditions. Mode energies of coal signal are mainly distributed in $0 \sim 1500 \mathrm{~Hz}$, which accounts for about 50\% of the total energy. Mode energies are mainly distributed in 3000 $\sim 5000 \mathrm{~Hz}$, which accounts for more than $50 \%$ of the total energy. The results indicate that the energy percentage of modal functions can be used as the characteristic index for the coal and rock character recognition at fully mechanized caving mining face. Then we used the PSO-SVM algorithm to do the classification, and the classification accuracy of this method is $90 \%$. The conclusion indicates that the method put forward can be well applied in realizing coal and rock identification.
\end{abstract}

Keywords: Coal and rock character recognition; Variational mode decomposition; characteristic extraction; PSO; SVM

\section{Introduction}

Fully mechanized top coal caving is a high-yielding and efficient way of thick seam mining. But the degree of drop of top coal and rock all rely on the artificial judgment, which easily lead to the problem of over caving and owing caving. And the problem leads to the decrease of the coal quality and potential security. So the coal- rock recognition is a key point to achieve accurate control of the degree of drop and increase the coal quality [1-2]. Recently years a lot of researchers worked on coal-rock recognition by analyzing the signal of coal and rock with several signal analysis methods, which include time domain statistical analysis, wavelet packet analysis [3] and empirical mode decomposition(EMD) [4-6] and so on. Zhang Yanli analyzed acoustic signals with EMD, and she found significant differences in the frequency distribution of modes between coal and rock ${ }^{[4]}$. WANG Baoping calculated the energy of obtained modes decomposed by EMD and she also found mode energies can distinguish coal and rock, and then use the energy to do the coal-rock recognition [5]. CONG Xiaoyan used EMD to analyzed the vibration signals of coal and rock, calculated the kurtosis of decomposed modes, and he resynthesized signal with the modes whose difference between coal and rock is biggest, then he did the recognition with Hilbert marginal spectrum of resynthesized signal [6].

Variational mode decomposition is a new non-recursive and self-adaptive signal processing method put forward by Dragomiretskiy and Dominique Zosso in 2014 [7]. In 
the process of obtaining the decomposition components, the frequency center and bandwidth of each component can be determined by iterative searching for the optimal solution of the variational mode, which can adaptively realize the frequency domain division and the effective separation of the components. Recursive variational mode decomposition model like EMD cannot correctly separate signal which has similar frequency components, and easily cause modes mixture when analyzing noisy signal or more complex signal. However, VMD algorithm has a solid theoretical foundation, better noise robustness, and can achieve more accurate signal separation. LIU Changliang put forward a method based on VMD and standard fuzzy $\mathrm{C}$ means clustering for fault diagnosis [8]. Yanxue Wang applied VMD in detecting rub-impact fault of the rotor system, and the effect is better that EWT, EEMD and EMD methods [9]. Abhay Upadhyay and Ram Bilas Pachori used the VMD method on the instantaneous detection of voiced/non-voiced (V/NV) regions in the speech signals and showed effectiveness compared to GCIs, MEDT and other methods [10].

Currently, VMD method has not been applied to the recognition problem of coal and rock, and VMD method is more suitable for analyzing complex signals compared with recursive variational mode decomposition method. This paper carried out the coal and rock caving experiment at Fully Mechanized Caving Mining Face and obtained the vibration signal of the hydraulic support tail beam under the different working conditions of coal falling and rock falling. VMD method was applied to analyze the signals and study the energy of the obtained modes, and energy percentages of modes were extracted as characteristic indexes of coal-rock recognition. In the end, setting EMD analysis and SVM as the comparative group, coal-rock classifier was designed combined with PSO-SVM classification algorithm.

\section{Variational Mode Decomposition}

In the VMD description, Intrinsic Mode Functions are amplitude-modulated-frequencymodulated (AM-FM) signals, written as:

$$
u_{k}(t)=A_{k}(t) \cos \left(\phi_{k}(t)\right)
$$

The phase $\phi_{k}(t)$ is a non-decreasing function, $\phi_{k}^{\prime}(t) \geq 0$ the envelope is non-negative, and the instantaneous frequency $\omega_{k}(t)=\phi_{k}^{\prime}(t)$.both the envelope $A_{k}(t)$ and $\omega_{k}(t)$ vary much slower than the phase. The problem of VMD is to find $\mathrm{k}$ modes, each being band-limited about a center frequency estimated on-line, to minimize the sum of the estimated bandwidths of all modes. And the constraint is that the sum of the modes is equal to the input signal $\mathrm{f}$.

For each mode $u_{k}(t)$, the associated analytic signal is obtained as $[\delta(t)+j /(\pi t)] * u_{k}(t)$ by means of Hilbert transform. Mix each associated analytic signals with exponential tuned to the respective estimated center frequency. Then estimate the bandwidth through the squared L2-norm of the gradient. The constrained variational problem is the following:

$$
\left\{\begin{array}{l}
\min _{\left\{u_{k}\right\},\left\{\omega_{k}\right\}}\left\{\sum_{k}\left\|\left[\left(\delta(t)+\frac{j}{\pi t}\right) * u_{k}(t)\right] e^{-j \omega_{k} t}\right\|^{2}\right\} \\
\text { s.t. } \sum_{k} u_{k}=f
\end{array}\right.
$$

Introduce quadratic penalty term $C$ and lagrangian multipliers $\lambda$ to render the problem unconstrained. The quadratic penalty term is to enforce strict data fidelity, the lagrangian multiplier is to enforce strict constrain. The augmented Lagrangian is as follow: 


$$
\begin{aligned}
& L\left(\left\{u_{k}\right\},\left\{\omega_{k}\right\}, \lambda\right)= \\
& \quad \alpha \sum_{k}\left\|\partial_{t}\left[\left(\delta(t)+\frac{j}{\pi t}\right) * u_{k}(t)\right] e^{-j \omega_{k} t}\right\|^{2}+\left\|f(t)-\sum_{k} u_{k}(t)\right\|{ }_{2}^{2}+\left\langle\lambda(t), f(t)-\sum_{k} u_{k}(t)\right\rangle
\end{aligned}
$$

VMD method use alternate direction method of multipliers (ADMM) algorithm to solve the above minimization problem, the modes and center frequencies are solved as:

$$
\begin{gathered}
\hat{u}_{k}^{n+1}(\omega)=\frac{\hat{f}(\omega)-\sum_{i \neq k} u_{i}(\omega)+\frac{\hat{\lambda}(\omega)}{2}}{1+2 \alpha\left(\omega-\omega_{k}\right)^{2}} \\
\omega_{k}^{n+1}=\frac{\int_{0}^{\infty} \omega\left|\hat{u}_{k}(\omega)\right|^{2} d \omega}{\int_{0}^{\infty}\left|\hat{u}_{k}(\omega)\right|^{2} d \omega}
\end{gathered}
$$

Where $\hat{\boldsymbol{u}}_{k}^{n+1}(\omega)$ is identified as a Wiener filtering of the current residual, with signal prior $^{1 /\left(\omega-\omega_{k}\right)^{2}}, \omega_{k}^{n+1}$ is the center of gravity of the corresponding mode's power spectrum.

\section{PSO-SVM}

\subsection{Support Vector Machine}

According to statistical theory and the structural risk minimization principle, support vector machine (SVM) is to find the optimum hyperplane (decision function) in the data space, and the hyperplane can distinguish different types of data as much as possible.

For sample set $\left(x_{i}, y_{i}\right), i=1,2, \cdots N, x_{i} \in R_{d}, y_{i} \in\{-1,+1\}, x_{i}$ is the eigenvalue, $y_{i}$ is its attribute values. The linear equations of Classification (That is linearly separable) is $f(x)=\omega \cdot x+b$, so we can get the following formula:

$$
\left.\begin{array}{ll}
\omega \cdot x_{i}+b \geq 0 & \text { if } y_{i}=1 \\
\omega \cdot x_{i}+b \leq 0 & \text { if } y_{i}=-1
\end{array}\right\} \Rightarrow y_{i}\left[\left(\omega \cdot x_{i}\right)+b\right]-1 \geq 0, i=1, \cdots N
$$

In the case of non-linear separable, add slack variable $\xi_{i}$, and the corresponding dual problem is

$$
y_{i}\left[\left(\omega \cdot x_{i}\right)+b\right]-1+\xi_{i} \geq 0, i=1, \cdots N
$$

With known data sets, there may be numerous hyperplane. The SVM method stipulates a hyperplane is the best hyperplane when the distance from the hyperplane to the closest data to the hyperlpane is the largest.

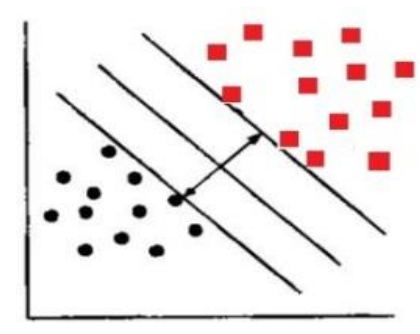

(a) Big Margin

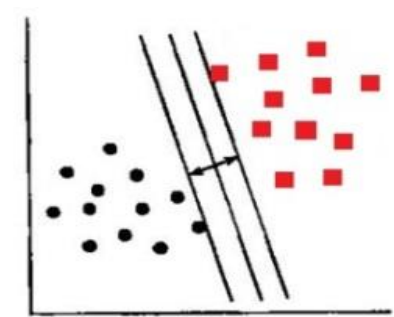

(b) Small Margin

Figure 1. Hyperplane of Classification 
The optimal hyperplane problem can be converted into quadratic optimization problem

$$
\left\{\begin{array}{l}
\min _{w, b} \frac{1}{2}\|w\|^{2}+C \sum_{i=1}^{n} \xi_{i} \quad \xi_{i}>0, i=1, \cdots N \\
\text { st. } \quad y_{i}\left[\left(w \cdot x_{i}\right)+b\right]-1 \geq 0
\end{array}\right.
$$

In the formula, $a_{i}$ is the $i$ th Lagrange multiplier. The solution of the $\omega_{\text {and }} b$ are

$$
\left\{\begin{array}{l}
\omega=\sum_{i=1}^{N} y_{i} \alpha_{i} x_{i} \\
b=y_{i}-\sum_{i=1}^{N} a_{i}\left(x_{i} \cdot x\right)
\end{array}\right.
$$

The corresponding decision function is

$$
f(x)=\operatorname{sgn}\left(\sum_{i=1}^{N} a_{i} y_{i}\left(x_{i}, x\right)+b\right)
$$

For separation surface, by using kernel function, SVM makes the feature vector to be mapped to a new feature space which is linearly separable and realizes the linear classification of samples in high dimensional feature space. Therefore, the corresponding decision function is

$$
f(x)=\operatorname{sgn}\left(\sum_{i=1}^{N} a_{i} y_{i} K\left(x_{i}, x\right)+b\right)
$$

$K\left(x_{i}, x_{i}\right)$ is called kernel function. Radial basis function (RBF) as a kind of kernel function is applied widely and well [11-12]. Under the appropriate parameters, SVM with $\mathrm{RBF}$ is always able to separate the data linearly in the feature space, and it will not make the space too complex [13], so set RBF as the kernel function.

\subsection{PSO-SVM}

The choice of parameters has a significant impact on the performance of support vector machine (SVM). The main parameters of SVM with RBF kernel function are kernel function parameter $g$ and penalty factor $C$, so the PSO-SVM method is optimize the parameters of the SVM by using particle swarm optimization algorithm, then design the support vector machine model with the optimized parameters.

Particle Swarm Optimization (PSO) is a kind of heuristic random optimization algorithm with strong global search ability and global optimization characteristics. In PSO, each potential solution is called a particle with no weight and no volume. The $i$ th particle flies in a D-dimensional search space to find the optimal solution, and The position and speed of the $i$ th particle is $X_{i}=\left[x_{i 1}, x_{i 2}, \ldots, x_{i d}\right]_{\text {and }} V_{i}=\left[v_{i 1}, v_{i 2}, \ldots, v_{i d}\right]$, where $x_{i d} \in\left[l_{d}, u_{d}\right]$, $d \in[1, D], \quad l_{d}$ and $u_{d}$ are the lower and upper bounds of the $d$ th dimension. The best previous position of any particle is recorded as the personal best called Pbest. The best solution visited by the whole swarm so far is the global best called ${ }^{\text {gbest }}$. The swarm is initialized with a population of random solutions. According to the indexes Pbest and 
the ${ }^{\text {gbest }}$, the algorithm searches for the best solution by updating particles' positions and velocities using the following formulae:

$$
\begin{gathered}
v_{i d}^{n+1}=\omega \times v_{i d}^{n}+c_{1} \times r_{1} \times\left(p_{i d}^{n}-x_{i d}^{n}\right)+c_{2} \times r_{2} \times\left(p_{g d}^{n}-x_{i d}^{n}\right) \\
x_{i d}^{n+1}=x_{i d}^{n}+v_{i d}^{n}
\end{gathered}
$$

$\omega$ is the inertia weight. ${ }^{c_{1}}$ and $c_{2}$ are acceleration constants. ${ }^{r_{1}}$ and $r_{2}$ are random values uniformly distributed in $[0,1] . P_{\text {id }}$ presents the Pbest ${ }_{\text {while }} P_{g d}$ presents the ${ }^{\text {gbest }}$.

In PSO-SVM, the optimizing function is classification accuracy of SVM, through continuous update gaussian radial basis kernel function parameter $\mathrm{g}$ and penalty factor $\mathrm{C}$ to get to the highest classification accuracy the best parameter values correspond to, then do the training and test of SVM classifier with obtained parameters. So the PSO-SVM algorithm process summary as shown in the Figure 2.

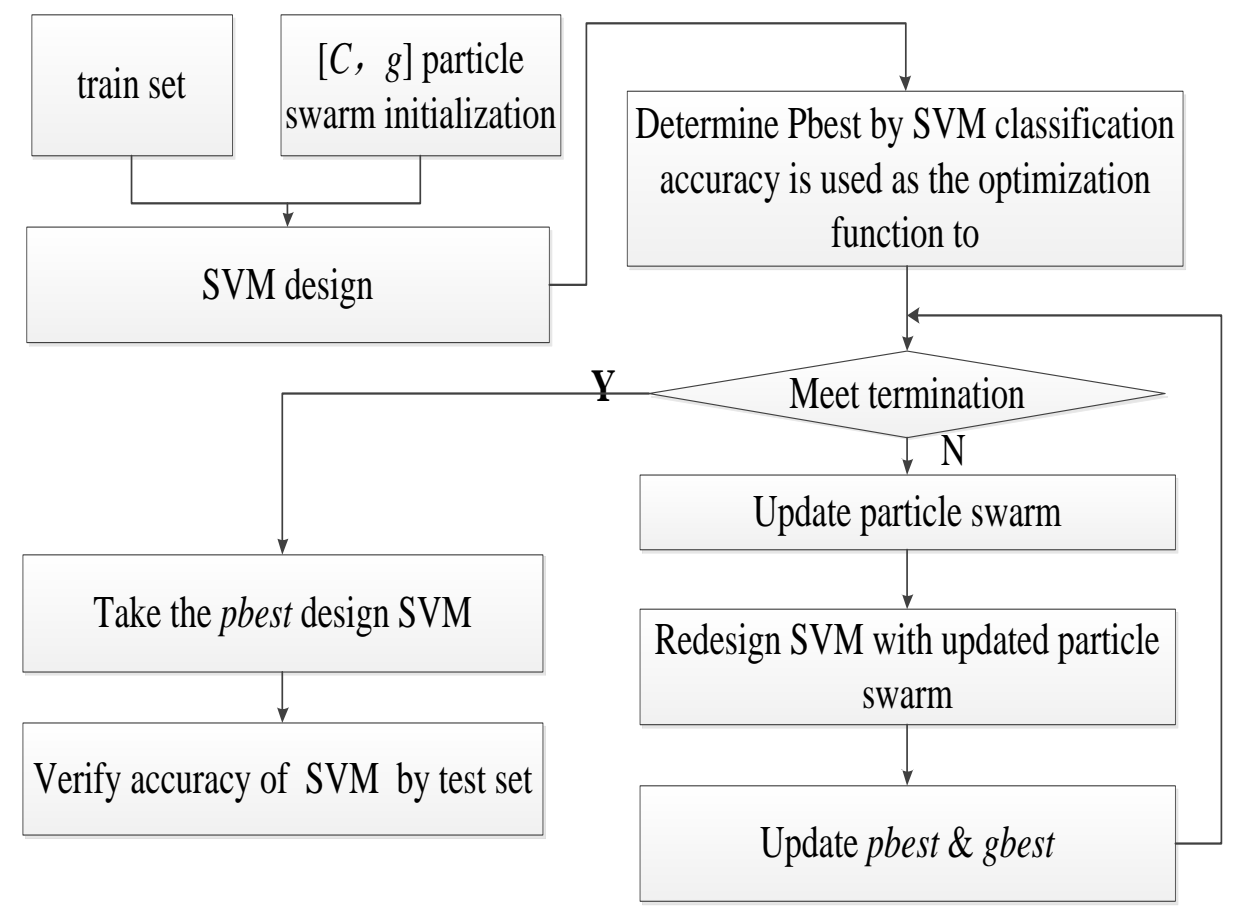

Figure 2. PSO-SVM Algorithm Flow Chart

\section{Top Coal and Rock Caving Experiment}

\subsection{Experiment Field and Equipment}

The experiment was carried out at the 9201working face of Danshuigou mine in Shuozhou city, Shanxi province. The 9201working face is the bringing back long wall face with lower position roof coal caving. The experiment was at Medium hard coal seam, where the dirt band was mudstone, and the Roof was Sandstone. The average slope of coal seam is $4^{\circ}$; the biggest height difference in the mining area is $16.5 \mathrm{~m}$. The equipment layout is shown in Figure3. 


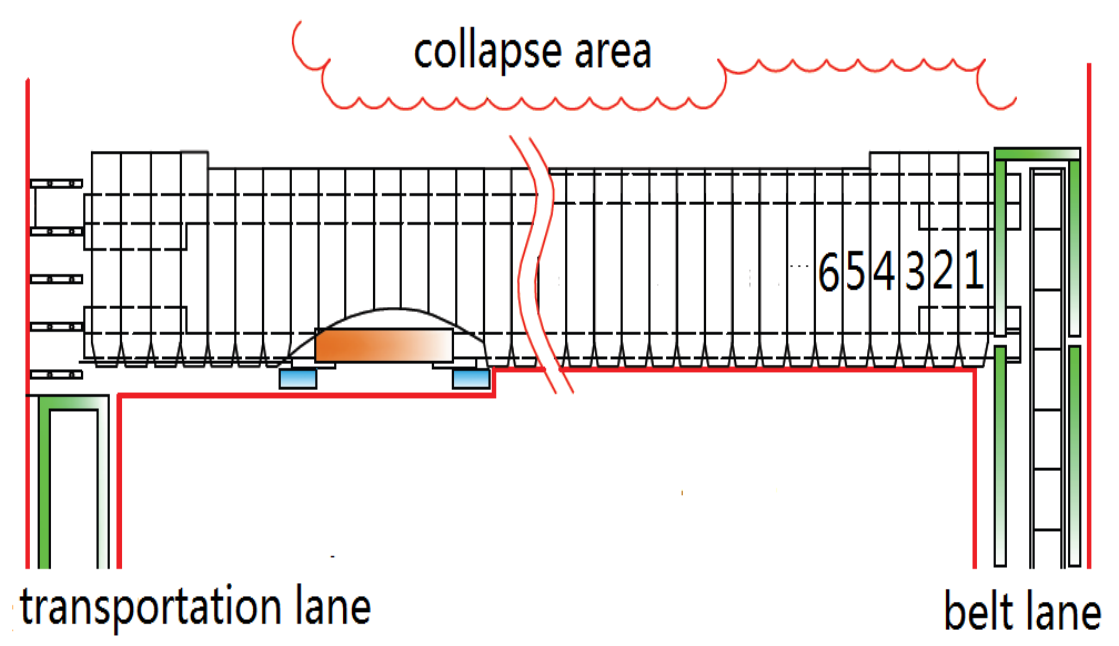

Figure 3. Equipment Layout of 9201 Workface

The experimental equipment mainly includes independent researched mine-used accelerometer and independent researched $\mathrm{YHJ}(\mathrm{C})$ mine portable digital recorder. The Accelerometer is shown in Figure 4(a), and can measure signals of multiple directions simultaneously by using multi-way magnetic suction base. The frequency range of the Accelerometer is $1 \sim 10 \mathrm{KHz}$ and the Sensitivity is $5.0 \mathrm{mV} /\left(\mathrm{m}^{\bullet} \mathrm{s}^{2}\right)$. The $\mathrm{YHJ}(\mathrm{C})$ mine portable digital recorder, as shown in Figure 4(b), is four-channel mine-used vibration signal equipment. The frequency range of the Accelerometer is $0 \sim 250 \mathrm{KHz}$.

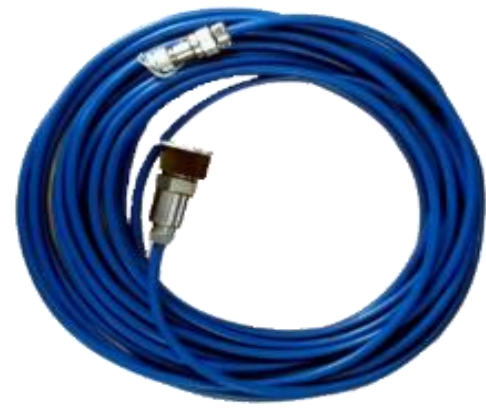

(a) Mine Accelerometer

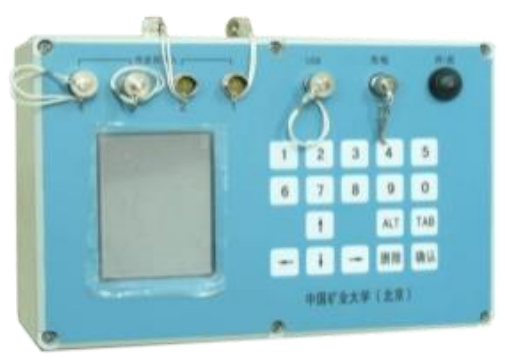

(b) $\mathrm{YHJ}(\mathrm{C})$ Mine Portable Vibration Recorder

Figure 4. Experimental Equipment

\subsection{Experimental Scheme}

The experiment was carried out at NO.39 hydraulic support at the working face. We arranged measuring points of vibration sensor in the inside of hydraulic support beam, and fixed the independent researched mine portable digital recorder at the hydraulic control panel, shown as Figure 5, which is easy for data collection and prevent the sensor from falling coal and rock. The sampling frequency is set to $10 \mathrm{kHz}$. When coal and rock collapse and impact hydraulic support, the Accelerometer obtains two kinds of shock vibration signals and the $\mathrm{YHJ}(\mathrm{C})$ mine portable digital recorder records the signal. The time-domain waveforms of the vibration signals obtained under different conditions are shown in Figure 6. 


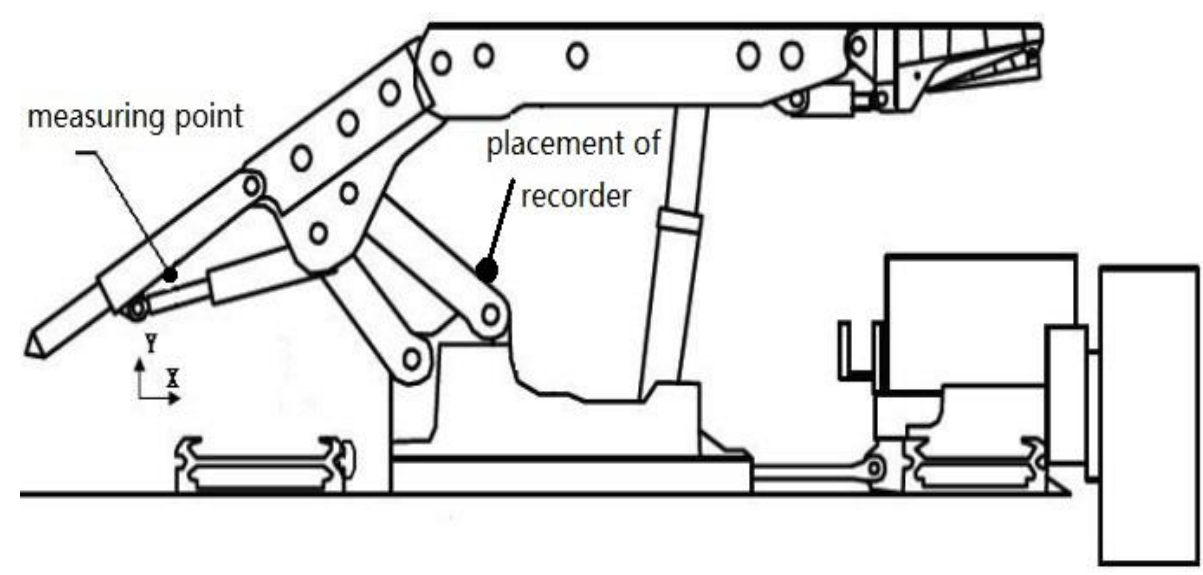

Figure 5. Schematic Diagram of Measurement Point

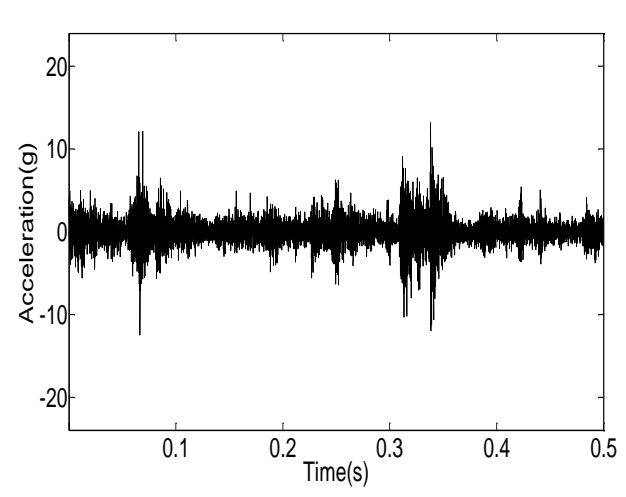

(1) Coal

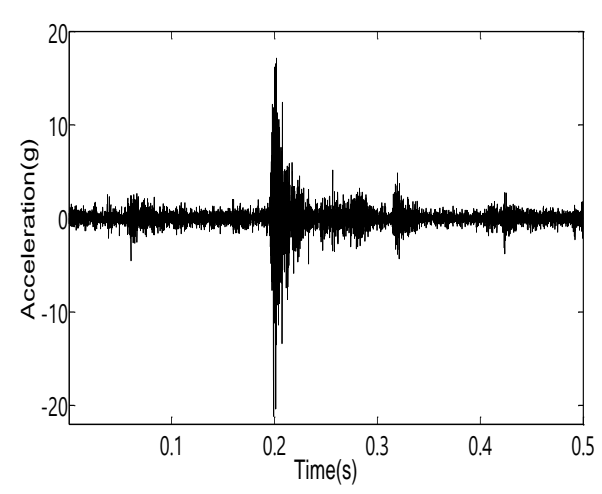

(2) Rock

Figure 6. Time Domain Waveform of Vibration Signal when Coal and Rock Falling

\section{Characteristic Extraction}

\subsection{Simulation}

Vibration signal obtained from experiment contains noise signal, presents discontinuity of frequency and other complex composition, so we choose noisy signal, intermittent signal and signal including square wave to do the VMD simulation analysis. The formula of the chosen noise signal is below:

$$
y=\cos (4 \pi t)+\cos (48 \pi t)+0.2 \cos (512 \pi t)+\eta(0, N)
$$

The analysis results are shown as in Figure7. In the Figure (a), the black dotted line indicates the real components of the input signal, and color solid line indicates the components decomposed by VMD method. After VMD analysis of noisy signal, all the frequency components are decomposed, and the decomposed noise part has a little deviation with original noise part but within acceptable range. The EMD analysis decomposes the original noisy signal into five modes, and there is an over-decomposition where mode2, mode 3 and mode 4 are supposed to be one mode. 


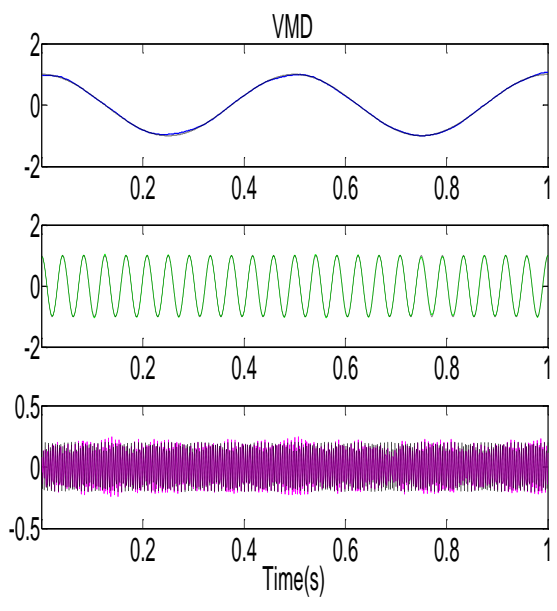

(a) VMD
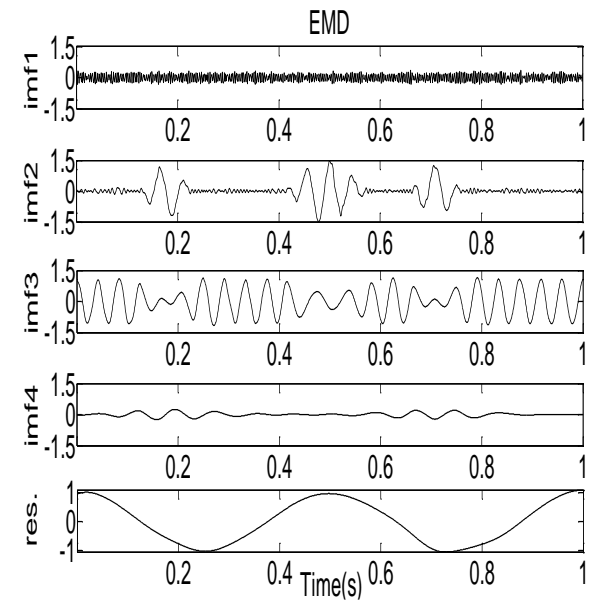

(b) EMD

\section{Figure 7. Simulation Results of Noise Signal by VMD and EMD}

Analyze signal of discontinuous frequency with VMD and EMD method. The formula is shown below.

$$
y=\cos (5 \pi t)+ \begin{cases}0.25 \cos (160 \pi t) & t \leq 0.5 \\ 0.1 \cos (250 \pi t) & t>0.5\end{cases}
$$

The analysis results are shown as in Figure 8. After VMD analysis of signal with discontinuous frequency, all the frequency components are accurately decomposed, EMD analysis cannot decompose the discontinuous frequency components into different modes where different parts are decomposed into one mode.

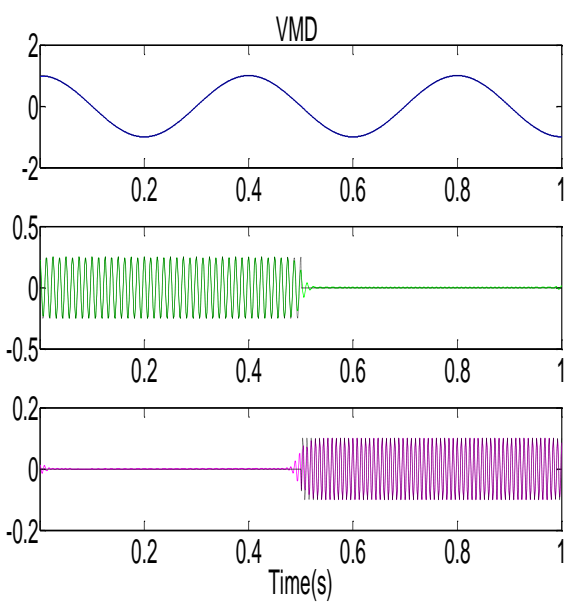

(a) VMD
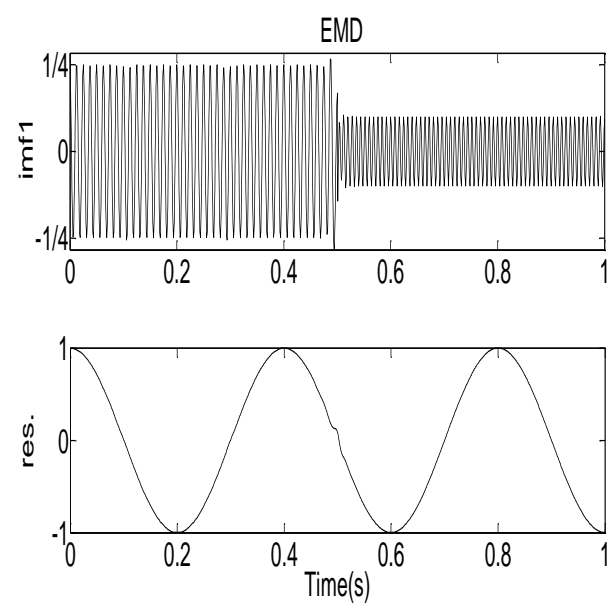

(b) EMD

Figure 8. Simulation Results of Intermittent Signal by VMD and EMD

Analyze signal including periodic square wave with VMD and EMD method. The formula is shown below.

$$
\begin{aligned}
& \mathrm{y}=\cos (5 \pi \mathrm{t})+0.25 \cos (160 \pi \mathrm{t})+\sum_{n=0}^{124} x(t-n \cdot T) \\
& x(t)=\left\{\begin{array}{ll}
0.1 & (0<\mathrm{t}<T / 2) \\
-0.1 & (-T / 2<\mathrm{t}<0)
\end{array}, T=\frac{1}{f}=\frac{1}{125} s\right.
\end{aligned}
$$

The analysis results are shown as in Figure 9. After VMD analysis all the frequency 
components are decomposed, but the decomposed square wave part has a little deviation of amplitude with original part. EMD analysis cannot decompose the discontinuous frequency components into different modes where mode 2 and mode 3 are supposed to be one mode.
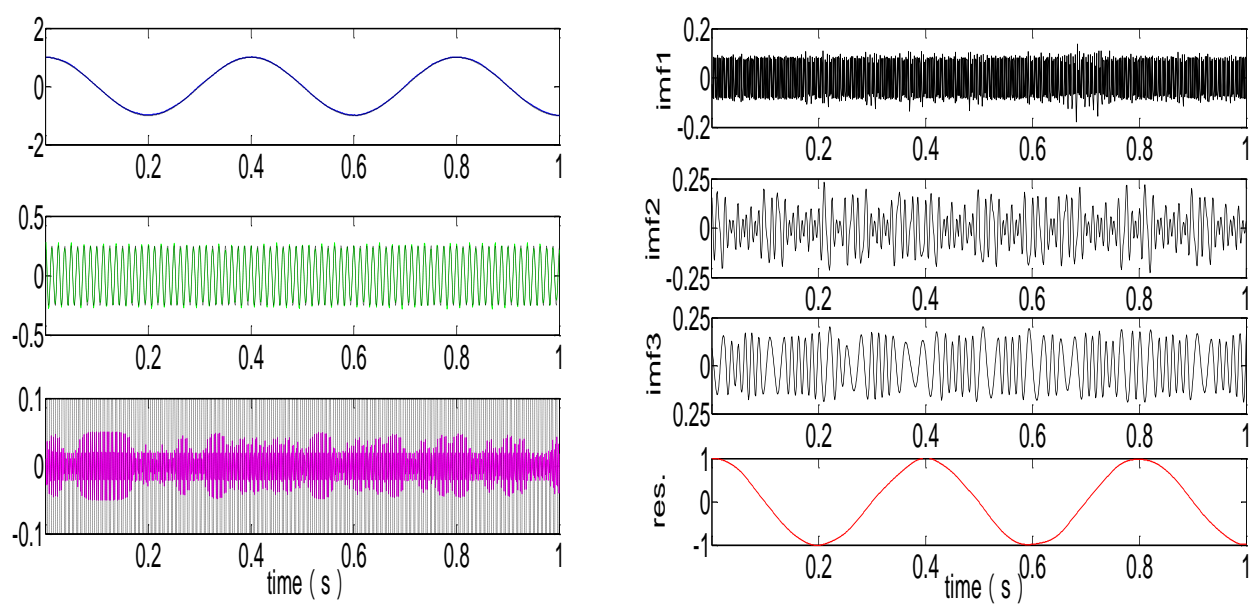

(a) VMD

(b) EMD

Figure 9. Simulation Results of Signal Including Square Wave by VMD and EMD

From the simulation analysis of three kinds of signal we know that VMD analysis method is more reliable for analyzing vibration signals obtained from experiment compared with EMD methods.

\subsection{Characteristic Analysis and Extraction}

The number of decomposed modes $K$, that is the main parameter of the VMD method, needs to be set in advance. In order to determine the value of $K$, we use one experimental signal to do the VMD analysis by setting different value of $K$, and the decomposed center frequencies are shown in Table 1.

Table 1. The Center Frequencies of the Modal Functions with Different $\mathrm{K}$

\begin{tabular}{|c|c|c|c|c|c|c|c|c|c|c|c|c|c|}
\hline & \multicolumn{6}{|c|}{$\mathrm{K}$} & \multicolumn{7}{|c|}{ Center frequencies $(\mathrm{Hz})$} \\
\hline 2 & 590 & 2089 & & & & & & & & & & & \\
\hline 3 & 590 & 2088 & 3451 & & & & & & & & & & \\
\hline 4 & 590 & 1406 & 2089 & 4292 & & & & & & & & & \\
\hline 5 & 588 & 1135 & 2089 & 4290 & 4630 & & & & & & & & \\
\hline 6 & 291 & 599 & 2071 & 3451 & 4298 & 4630 & & & & & & & \\
\hline 7 & 290 & 587 & 2061 & 2433 & 3438 & 4301 & 4630 & & & & & & \\
\hline 8 & 288 & 580 & 1136 & 2081 & 2434 & 3434 & 4305 & 4630 & & & & & \\
\hline 9 & 287 & 586 & 1135 & 1569 & 2077 & 2428 & 3442 & 4296 & 4630 & & & & \\
\hline 10 & 286 & 590 & 807 & 1133 & 1579 & 2081 & 2431 & 3449 & 4291 & 4630 & & & \\
\hline 11 & 286 & 585 & 812 & 1140 & 1570 & 2085 & 2426 & 3084 & 3436 & 4290 & 4630 & & \\
\hline 12 & 286 & 582 & 810 & 1135 & 1577 & 2080 & 2426 & 3084 & 3436 & 3802 & 4292 & 4630 & \\
\hline 13 & 286 & 582 & 806 & 1132 & 1582 & 2092 & 2150 & 2426 & 3084 & 3436 & 3802 & 4287 & 4630 \\
\hline
\end{tabular}

From Table 1 we can see that when $K=13$, there are two different modes with close center frequencies. We say that there is an over-decomposition [8]. Therefore, for all the obtained experimental signals, we set the value of modes $\mathrm{K}$ to be 12 .

Due to the physical properties of coal and rock, such as density and hardness, have obvious difference, the vibration signals of the hydraulic support tail beam impacted by falling coal and falling rock exist obvious difference in the frequency distribution [5]. 
Therefore, we intent to analyze experimental vibration signal with VMD method, then calculate energy and energy percentage of obtained modes with the formula (16) and formula (17), where $\mathrm{Ei}$ is the energy of modal function the ith center frequency corresponds to, $\mathrm{Pi}$ is the energy percentage of modal function the ith center frequency corresponds to over the total energy.

$$
\begin{aligned}
& E=\lim _{T \rightarrow \infty} \int_{-T}^{T}|x(t)|^{2} d t \\
& p_{i}=E_{i} / \sum_{i=1}^{12} E_{i}
\end{aligned}
$$

Choose signals of falling coal and rock to do the VMD analysis and each signal is decomposed into twelve modes with center frequency. Calculate the energies of all modes of coal and rock, and calculate the percentage of each energy value to the total energy value, the results are shown in Table 2.

Table 2. Center Frequencies, Energies and Energy Percentages of the Modes when Coal Falling and Rock Falling

\begin{tabular}{ccccccc}
\hline \multirow{2}{*}{ mode } & \multicolumn{3}{c}{ Coal } & \multicolumn{3}{c}{ Rock } \\
\cline { 2 - 7 } & $\begin{array}{c}\text { center } \\
\text { frequency }(\mathrm{Hz})\end{array}$ & energy & $\begin{array}{c}\text { energy } \\
\text { percentage }\end{array}$ & $\begin{array}{c}\text { center } \\
\text { frequency }(\mathrm{Hz})\end{array}$ & energy & $\begin{array}{c}\text { energy } \\
\text { percentage }\end{array}$ \\
\hline mode1 & 286 & 1863 & 14.1 & 151 & 113 & 0.9 \\
mode2 & 582 & 3025 & 22.8 & 591 & 107 & 0.8 \\
mode3 & 810 & 4462 & 3.4 & 1276 & 248 & 1.9 \\
mode4 & 1135 & 1005 & 7.6 & 1724 & 315 & 2.4 \\
mode5 & 1577 & 1865 & 14.1 & 2065 & 422 & 3.3 \\
mode6 & 2080 & 960 & 7.2 & 2357 & 538 & 4.2 \\
mode7 & 2426 & 599 & 4.5 & 2582 & 1794 & 13.9 \\
mode8 & 3084 & 5276 & 4 & 3076 & 1086 & 8.4 \\
mode9 & 3436 & 6433 & 4.9 & 3449 & 2967 & 22.9 \\
mode10 & 3802 & 656 & 4.9 & 3651 & 1520 & 11.8 \\
mode11 & 4292 & 863 & 6.5 & 4197 & 2119 & 16.3 \\
mode12 & 4630 & 795 & 6 & 4613 & 1702 & 13.2 \\
\hline
\end{tabular}

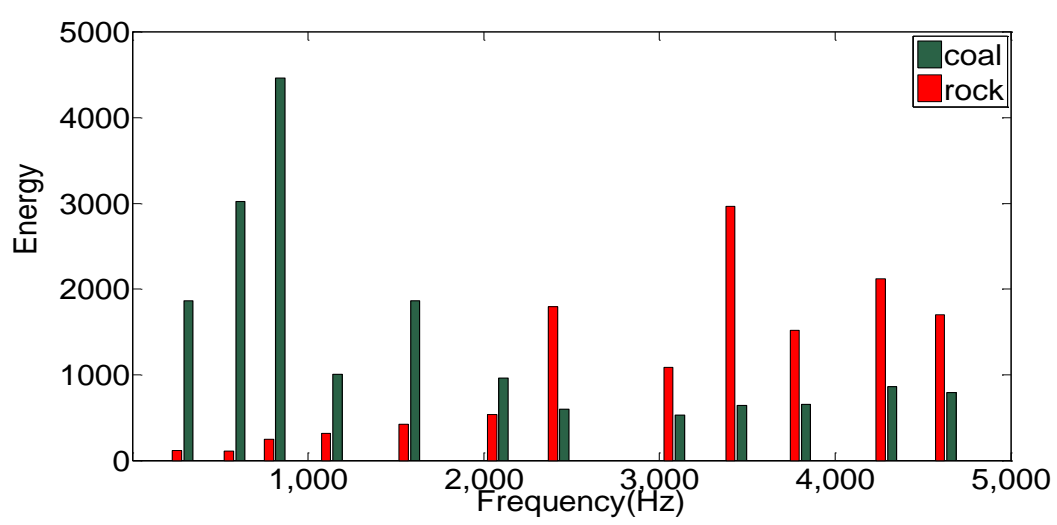

(a) Energies of Modes 


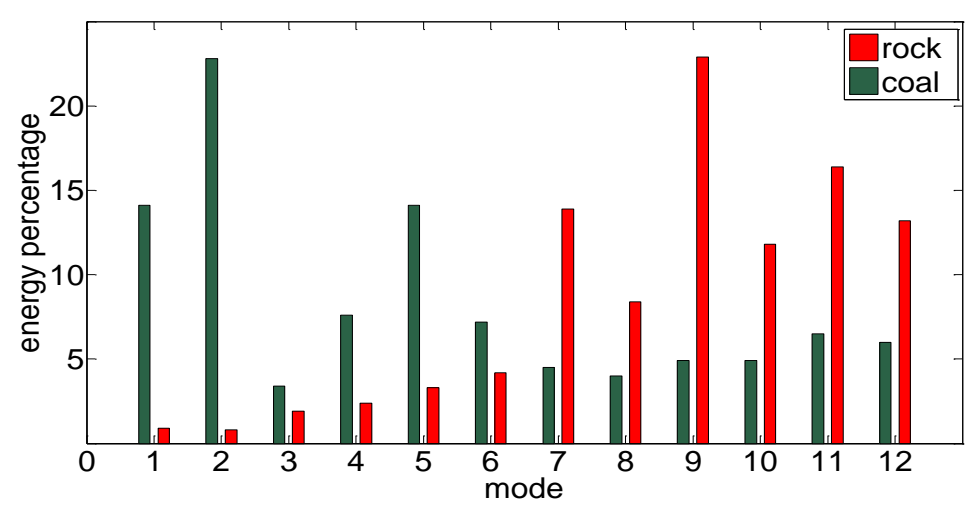

(b) Energy Percentages of Modes

\section{Figure 10. Energies and Energy Percentages of the Modals Function}

The Table 2 and Figure 10 show that the modes energies of coal signal are mainly distributed at the first some modes with small center frequency, the energy of modes below the frequency of $1500 \mathrm{~Hz}$ accounts for about $50 \%$ of the total energy. The modes energies of rock signal are mainly distributed at the back some modes with big center frequency, the energy of modes in the range of $3000 \sim 5000 \mathrm{~Hz}$ accounts for about $50 \%$ of the total energy, and the energy of modes below the frequency of $1500 \mathrm{~Hz}$ accounts for less than $10 \%$. The energy percentage of each mode can distinguish falling coal and falling rock, so we choose the energy percentages as the characteristic index for the coal and rock recognition.

Set the EMD analysis as the comparative group. Choose signals of falling coal and rock to do the VMD analysis separately and calculate the energies of all modes of coal and rock, the results are shown in Table 3 and Figure 11. The Table 3 and Figure 11 show that the mode energies of coal and rock decrease with increased modes. But there are also differences between coal and rock, the mode energies of rock decrease with increased modes more rapidly, and the mode energies of rock decrease with increased modes slower.

Table 3. Energies of the Modal Functions with EMD

\begin{tabular}{ccc}
\hline mode & coal & rock \\
\hline mode1 & 6220.75 & 20462.47 \\
mode2 & 2576.16 & 2913.9 \\
mode3 & 5893.78 & 909.23 \\
mode4 & 552.29 & 337.05 \\
mode5 & 1522.93 & 136.27 \\
mode6 & 675.22 & 166.64 \\
mode7 & 347.21 & 146.86 \\
mode8 & 231.57 & 38.67 \\
mode9 & 19.74 & 11.54 \\
mode10 & 137.42 & 8.36 \\
mode11 & 37.43 & 6.8 \\
mode12 & 23.78 & 3.83 \\
mode13 & 24.07 & 0.86 \\
\hline
\end{tabular}




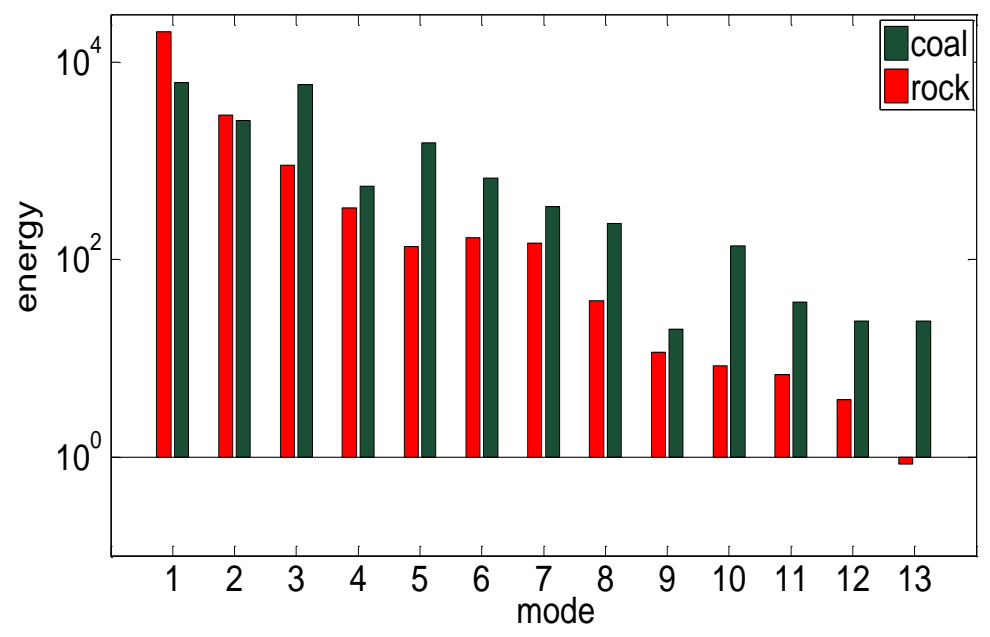

(a) Energies of Modes (Logarithmic Coordinate)

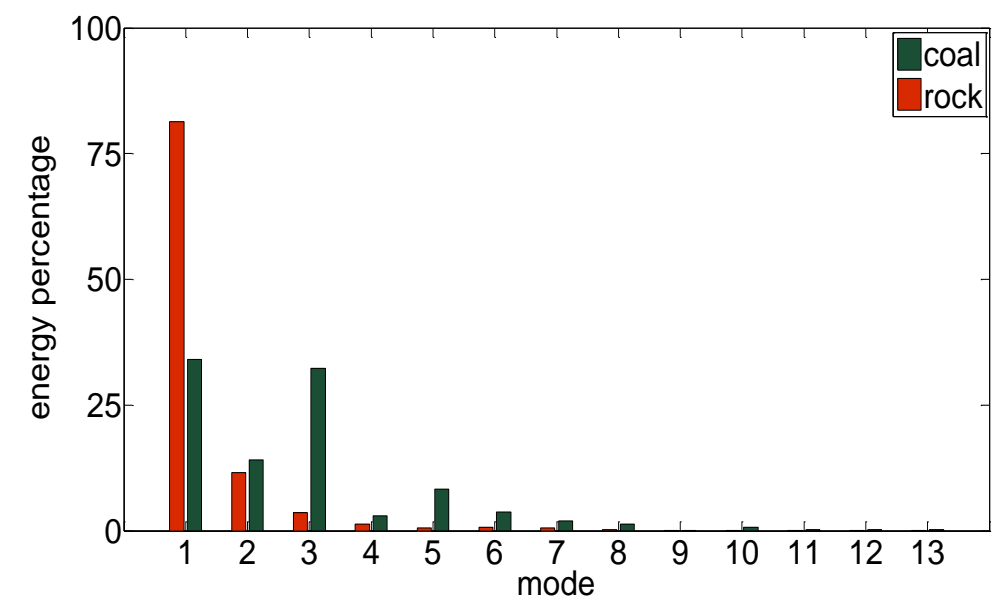

(b) Energy Percentages of Modes

Figure 11. Energies and Energy Percentages of the Modals Function

\section{Recognition}

Select 40 coal signals and 40 rock signals and set the parameter $K$ as 12 , analyze the signals with VMD and EMD method separately. Calculate the energies of each mode of coal and rock, and calculate the percentage of each energy value to the total energy. We take the energies of modes as the characteristic index to establish the characteristic vector and we get 40 coal characteristic vectors and 40 rock characteristic vectors. Take 25 coal characteristic vectors and 25 rock characteristic vectors as the train set to do the training of PSO-SVM, and then use the rest vectors to do the test. Meanwhile we choose the SVM algorithm as the comparative group. The results are shown in Table 4.

The Table 4 shows that the classification accuracy of PSO-SVM is higher than that of standard SVM whether using mode energy percentages of EMD or EMD as the characteristic index. In addition, the classification accuracy of VMD analysis is higher than that of EMD analysis whether using PSO-SVM or SVM algorithm. The classification accuracy is $90 \%$ when use VMD analysis and PSO-SVM classification algorithm which means that we can recognize coal and rock by combining VMD analysis and PSO-SVM algorithm. 
Table 4. Results of Train and Test

\begin{tabular}{|c|c|c|c|c|c|c|}
\hline \multicolumn{2}{|c|}{ method } & \multirow{2}{*}{$\begin{array}{l}\text { Best C } \\
10.43\end{array}$} & \multirow{2}{*}{$\begin{array}{l}\text { Best g } \\
3.11\end{array}$} & \multirow{2}{*}{$\begin{array}{c}\begin{array}{c}\text { Misclassification } \\
\text { number/accuracy } \\
\text { (coal) }\end{array} \\
1 / 93.3 \%\end{array}$} & \multirow{2}{*}{$\begin{array}{c}\begin{array}{c}\text { Misclassification } \\
\text { number /accuracy } \\
\text { (rock) }\end{array} \\
2 / 86.6 \%\end{array}$} & \multirow{2}{*}{$\begin{array}{c}\begin{array}{c}\text { Total } \\
\text { accurac } \\
y\end{array} \\
90 \%\end{array}$} \\
\hline V & $\begin{array}{l}\text { PSO- } \\
\text { SVM }\end{array}$ & & & & & \\
\hline D & SVM & - & - & $2 / 86.6 \%$ & $3 / 80 \%$ & $83.3 \%$ \\
\hline $\begin{array}{l}E \\
M\end{array}$ & $\begin{array}{l}\text { PSO- } \\
\text { SVM }\end{array}$ & 0.01 & 36.57 & $4 / 60 \%$ & $2 / 86.6 \%$ & $80 \%$ \\
\hline D & SVM & - & - & $8 / 60 \%$ & $4 / 80 \%$ & $60 \%$ \\
\hline
\end{tabular}

\section{Conclusions}

Aiming at the problem of "over caving" or "under caving" at fully mechanized caving mining face, we put forward a new method based on VMD analysis and PSO-SVM algorithm for coal and rock recognition, and the main results and conclusions are as follows:

(1)We carried out the top coal and rock caving experiment at fully mechanized caving mining face and obtained vibration signals of falling coal and rock.

(2) VMD and PSO-SVM are introduced and the reliability of the VMD algorithm is proved by simulation signal. The parameter of modes of VMD for the obtained signals is determined.

(3) Extract the energy percentage of the decomposed modes by VMD as the characteristic index. The energy of modes of coal signal is mainly distributed in the range of $0 \sim 1500 \mathrm{~Hz}$, which account for about $50 \%$ of the total energy. The energy of modes of coal signal is mainly distributed in the range of $3000 \sim 5000 \mathrm{~Hz}$, which account for about $50 \%$ of the total energy.

(4)VMD and PSO-SVM were combined to do the coal and rock recognition, the classification accuracy is 90\%. EMD analysis and SVM algorithm were also used as the comparative group, and it turned out that the classification accuracy is highest when VMD and PSO-SVM were applied.

\section{References}

[1] Z. Xin, W. Zhengxiang, W. Chengguang, "Research on Coal-rock Interface Recognition Technology", Coal Mine Machinery, vol. 36, no. 2, (2015), pp. 3-5.

[2] Z. Ningbo, L. Yan, L. Changyou, "Basic study on automatic detection of coal and gangue in the fully mechanized top coal caving mining", Journal of Mining and Safety Engineering, vol. 31, no. 4, (2014), pp. 532-536.

[3] Z. Shuanfeng, "Coal-rock interface recognition based on multi wavelet packet energy", Journal of Xi'an University of Science and Technology, vol. 9, no. 5, (2009), pp. 584-588.

[4] W. Baoping, W. Zengcai, Z. Wanzhi, "Coal-rock Interface Recognition Method Based on EMD and Neural Network", Journal of Vibration, Measurement \& Diagnosis, vol. 32, no. 4, (2012), pp. 586-590.

[5] Y. Zhang, S. Zhang, "Identification of coal and rock interface based on empirical mode decomposition", Coal Technology, vol. 9, (2007), pp. 49-51.

[6] X. Cong, Z. Wang, B. Wang, "Application of filtering method based on EMD and kurtosis in coal - rock interface recognition", Journal of Vibration, Measurement \& Diagnosis, (2015).

[7] K. Dragomiretskiy, D. Zosso, "Variational mode decomposition, IEEE Trans. Signal Process", vol. 62, no. 3, (2014), pp. 531-544.

[8] C. Liu, Y. Wu, C. Zhen, "Rolling Bearing Fault Diagnosis Based on Variational Mode Decomposition and Fuzzy C Means Clustering”, Proceeding of the CSEE, vol. 35, no. 13, (2015), pp. 3358-3365.

[9] W. Yanxue, M. Richard, X. Jiawei, "Research on variational mode decomposition and its application in detecting rub-impact fault of the rotor system", Mechanical Systems and Signal Processing, (2015), pp. 243-251. 
[10] Instantaneous voicednon-voiced detection in speech signals based on variational mode decomposition

[11] S. Zhu, R. Zhang, "Research for Selection of Kernel Functions Used in Support Vector Machine", Science Technology and Engineering, vol. 8, no. 16, (2008), pp. 4513-4517.

[12] J. Pang, X. Li, J. Xie, "Microarchitectural design space exploration via support vector machine", Acta Scientiarum Naturalium Universitatis Pekinensis, (2010), vol. 46, no. 1, pp. 55-63.

[13] H. Rong, G. Zhang, W, Jin, "Selection of Kernel Functions and Parameters for Support Vector Machines in System Identification", Journal of System Simulation, vol. 11, no. 5, (2006).

[14] W. Xiong, B. Xu, "Study on Optimization of SVR Parameters Selection Based on PSO", Journal of System Simulation, (2006), vo. 9, pp. 2442-2445.

[15] Y. Yun, "Human Face Recognition Based on PSO and BP Neural Network", Computer Simulation, vol. 25, no. 8, (2008), pp. 201-204.

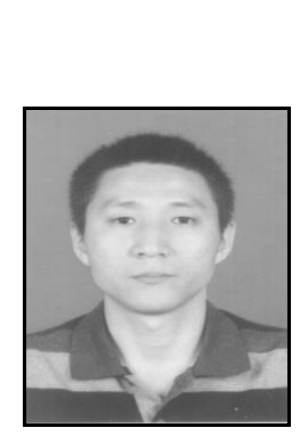

\section{Author}

Xue Guang-Hui, Mechanical and Electronic Engineering Doctor, now is an Associate professor, chairman of the Measurement and Control Technology and Instrumentation Program Department of China University of Mining and Technology (Beijing). He got his B Sc in Communication Engineering, M Sc in Mechanical Design and Theory, Ph.D. in Mechanical and Electronic Engineering. Dr. Xue Guang-Hui got the Scientific Improvement Award of China Coal Industry Association twice. Currently Dr. Xue Guang-Hui's research focuses on the Coal-Rock Identification, Signal Analysis and Processing, Data Acquisition and Processing and Development of Instrument pertinent to measurement and gauging. 